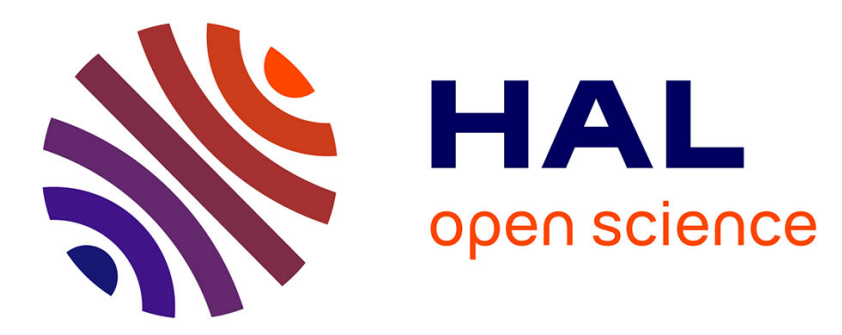

\title{
Upgrading linear to sliding mode feedback algorithm for a digital controller
}

\author{
Gabriele Perozzi, Andrey Polyakov, Felix Miranda-Villatoro, Bernard \\ Brogliato
}

\section{- To cite this version:}

Gabriele Perozzi, Andrey Polyakov, Felix Miranda-Villatoro, Bernard Brogliato. Upgrading linear to sliding mode feedback algorithm for a digital controller. CDSC 2021: 60th IEEE Conference on Decision and Control, Dec 2021, Fairmont Austin, United States. pp.1-6. hal-03306173

\section{HAL Id: hal-03306173 \\ https://inria.hal.science/hal-03306173}

Submitted on 28 Jul 2021

HAL is a multi-disciplinary open access archive for the deposit and dissemination of scientific research documents, whether they are published or not. The documents may come from teaching and research institutions in France or abroad, or from public or private research centers.
L'archive ouverte pluridisciplinaire HAL, est destinée au dépôt et à la diffusion de documents scientifiques de niveau recherche, publiés ou non, émanant des établissements d'enseignement et de recherche français ou étrangers, des laboratoires publics ou privés. 


\title{
Upgrading linear to sliding mode feedback algorithm for a digital controller
}

\author{
Gabriele Perozzi, Andrey Polyakov, Félix Miranda-Villatoro, Bernard Brogliato
}

\begin{abstract}
The goal of this paper is to investigate if it is possible to upgrade a given linear controller to a sliding mode one with an improvement of the control performance. Starting from a given linear controller, a design procedure for a sliding mode control having better performance than the linear one, is proposed. If the system has disturbances, which is always the case in experiments, the upgraded sliding mode control brings also a better robustness with respect to the given linear robust controller. The main idea is to divide the state-space into two areas, introducing a design parameter which separates the area of the linear control from the area of the sliding mode control. Some issues related to the chattering reduction are discussed. The control scheme's efficiency is demonstrated experimentally on a rotary inverted pendulum. The experimental results demonstrate the effectiveness of the obtained controls, and show an improvement with respect to the given linear proportional control.
\end{abstract}

\section{INTRODUCTION}

Sliding Mode Control (SMC) is a popular nonlinear setvalued control law, which is, theoretically, nonsensitive with respect to the so-called matched perturbations and, consequently, guarantees better control precision [1],[2], [3]. However, the chattering phenomenon does not allow the ideal sliding mode to be realized in practice. This may imply a degradation of the control precision instead of the promised improvement. Several methods for the chattering analysis and chattering reduction can be found in the literature, depending on its origin (unmodelled dynamics, discretization method) [1], [4], [5], [6], [7], [8]. However, the chattering problem is still of interest [9], [10].

On the other hand, a linear controller is the most popular industrial solution. It is supported with many methods of control parameters tuning in both frequency and time domains (see, e.g., [11],[12]). The linear control provides a satisfactory performance in many real applications. In this paper we try to answer the following question:

Is it possible to upgrade (transform) a well-tuned linear controller to sliding mode one with a guaranteed improvement of the control precision?

More precisely, we want to design a sliding mode control algorithm, which quality can not be worse than the quality of the linear feedback.

The method presented in this paper assumes that a linear stabilizing feedback is already designed for the system. It

G. Perozzi and A. Polyakov are with Univ. Lille, INRIA, Centrale Lille, CNRS, UMR 9189 - CRIStAL, F-59000 Lille, France.

F. Miranda Villatoro and B. Brogliato are with Univ. Grenoble Alpes, INRIA, CNRS, LJK, Grenoble INP, 38000 Grenoble, France.

Corresponding author e-mail: gabriele.perozzi@inria.fr

This work was funded by the project ANR DigitSlid ANR-18-CE400008-01. is modified only in a certain zone of the state space, and a sliding mode algorithm is derived. Since the sliding mode control is obtained from a linear one, it does not need a selection of all SMC parameters. For the chattering reduction, the conventional trick which consists of replacing the signfunction by a linear saturation function is chosen [1]. The parameter of the saturation function usually is tuned for the concrete application. In our case, we introduce a oneparameter family of the nonlinear control laws, which has sliding mode and linear algorithms as limit cases. Hence, the procedure of the upgrade can be formulated as follows: starting from the linear (already well-tuned) feedback we modify the mentioned scalar parameter and approach the sliding mode case as long as the control quality is improving. It is obvious that such a scheme prevents the degradation of the control quality during the upgrade.

The problem to be discussed is formulated in Section II. The upgrading procedure to design a SM control starting from linear controller is presented in Section III. Then, the Section IV presents the digital implementation procedure and the consequent chattering reduction of SMC. The experiments are illustrated in Section V, and the final Section VI concludes the paper.

\section{Problem Statement}

Let us consider the problem of stabilization of the linear plant

$$
\dot{x}=A x+B u+f(t, x), \quad t \geq 0,
$$

where $x(t) \in \mathbb{R}^{n}$ - the system state, $u(t) \in \mathbb{R}$ - the control input, $A \in \mathbb{R}^{n \times n}$ and $B \in \mathbb{R}^{n \times 1}$ are system matrices assumed to be known, and the function $f: \mathbb{R} \times \mathbb{R}^{n} \rightarrow \mathbb{R}^{n}$ describes the system's uncertainties and disturbances.

The whole state $x$ is assumed to be available (measured or estimated), the pair $\{A, B\}$ is controllable. We assume that the system is already controlled by a linear feedback

$$
u_{\text {lin }}=K_{\text {lin }} x, \quad K_{\text {lin }} \in \mathbb{R}^{1 \times n} .
$$

Various schemes are developed for tuning of the control gain $K_{\text {lin }}$ (see e.g. [11]). We assume that $K_{\text {lin }}$ is already well-tuned (e.g. optimally tuned in time or frequency domains) such that it stabilizes at least the linear (unperturbed, $f(t, x)=0$ ) system (1) at zero.

Assumption 1: Assume that the matrix of the closed-loop linear system $A+B K_{\text {lin }}$ is Hurwitz and it has at least one real eigenvalue $\lambda<0$ such that the corresponding eigenvector $v \in \mathbb{R}^{1 \times n} \neq 0$ :

$$
v\left(A+B K_{\text {lin }}\right)=\lambda v
$$


is not orthogonal to $B$ :

$$
v B \neq 0 \text {. }
$$

On the one hand, it is well-known (see, e.g. [13]) that the linear controller is robust with respect to some Lipschitz nonlinearities. For example, the system (1), (2) remains globally asymptotically stable provided that $f$ satisfies the inequality

$$
\|f(t, x)\| \leq \eta\|x\|, \quad \forall t \in \mathbb{R}, \forall x \in \mathbb{R}^{n}
$$

and $\eta \geq 0$ is sufficiently small. The latter means that $f$ has to vanish at $x=0$.

On the other hand, a SMC steers all solutions of the closedloop system to a sliding surface $C x=0$ in a finite time [1], [3], where $C \in R^{1 \times n}$. Theoretically, it stabilizes the origin of the system (1) and completely rejects the so-called matched perturbations, while the mismatched perturbations satisfy the same assumption (5). The mentioned condition is formalized by the following inequality:

$$
\|f(t, x)-B C f(t, x)\| \leq \eta\|x\|, \quad \forall t \in \mathbb{R}, \forall x \in \mathbb{R}^{n},
$$

where $\eta \geq 0$ is sufficiently small and the row vector $C \in R^{1 \times n}$ defines a sliding surface $C x=0$ of a SMC system, $C B=1$.

The inequality (6) means that the function $f$ may have non-vanishing components at $x=0$, which belong to range $B$ (matched perturbations). Therefore, the sliding mode control indeed may reject a larger class of perturbations.

Since, in practice, the function $f$ is usually unknown, the quality of both controllers can only be validated with real experiments. In practice, due to the chattering phenomenon, the implementation of a sliding-mode algorithm may not guarantee a better quality of regulation than the linear one. The main goal of this work is to develop a method for upgrading a linear controller to a sliding mode one with an improved control quality. If the system has disturbances, which is always the case in experiments, the upgraded SMC brings also a better robustness with respect to the given linear robust controller.

\section{SLIDING MODE CONTROL DESIGN FROM A LINEAR FEEDBACK LAW}

The classical concept of the sliding-mode control design for the linear plant (1) is the two step procedure [1], [2], [3]:

1) select the so-called sliding surface $C x=0$ such that the motion of the considered system on this surface is stable (all trajectories converge to zero when time goes to infinity);

2) define a control law which steers the state of (1) towards the surface $C x=0$ in a finite time and ensures that this surface is a positively invariant set of the system (see, e.g. [1] for the rigorous mathematical definition of the sliding mode).

The sliding-mode control law is given by

$$
u_{S M}=K_{n o m} x+\gamma(t, x) \operatorname{sign}(C x),
$$

where $\gamma: \mathbb{R} \times \mathbb{R}^{n} \rightarrow(-\infty, 0)$ is such that $\inf \gamma(t, x)<0$,

$$
K_{\text {nom }}=-(C B)^{-1} C A \text {, }
$$

the row vector $C \in R^{1 \times n}$ is selected such that $C B \neq 0$ and the differential-algebraic equation

$$
\left\{\begin{array}{l}
\dot{x}=\left(I_{n}-B(C B)^{-1} C\right) A x, \\
C x=0,
\end{array}\right.
$$

is globally asymptotically stable. Also the signum function is defined as: $\operatorname{sgn}(\sigma)=-1$ if $\sigma<0, \operatorname{sgn}(\sigma)=1$ if $\sigma>0$, and $\operatorname{sgn}(0)=[-1,1]$.

Such a selection of an equivalent-control-based algorithm together with the above assumptions guarantees that the differential inclusion

$$
\dot{\sigma} \in \gamma(t, x) \operatorname{sgn}(\sigma)+C f(t, x), \quad \sigma=C x,
$$

holds, securing that the surface $C x=0$ is a finite-time attractive invariant manifold of the closed-loop system (1), (7) provided that $\gamma(t, x)+|C f(t, x)|<0$ for all $t \in \mathbb{R}$ and all $x \in \mathbb{R}^{n}$.

In the case analysed here, a linear feedback (2) is already well-tuned and provides a good performance for the closed-loop system. The objective is to make a "minimal" modification of the linear feedback in order to transform it to a sliding-mode control algorithm (7) and consequently to improve the control quality.

Since, by Assumption 1, the matrix of closed-loop linear system $A+B K_{\text {lin }}$ is Hurwitz and has a real eigenvalue $\lambda<0$, then the corresponding eigenvector $v \in \mathbb{R}^{1 \times n}$ defines an invariant manifold $v x=0$ of the linear closed-loop system. This manifold is a sort of sliding surface of the linear unperturbed system, but without the finite-time attraction property. Therefore, for the sliding-mode control (7) the sliding surface

$$
C x=0, \quad C=\frac{v}{v B} .
$$

is selected.

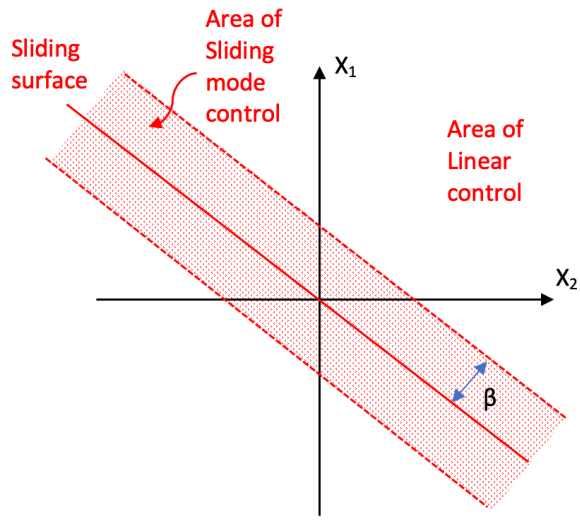

Fig. 1. The main idea of the upgrade from Linear to SMC.

The main idea of this work is to define the sliding mode control (7) such that (see Fig. 1)

$$
u_{S M}(x)=K_{l i n} x \quad \text { for }|C x| \geq \beta,
$$

and for $|C x|<\beta$ the sliding mode algorithm has the form (7), which steers all trajectories of the closed-loop system to the 
surface $|C x|=0$ in a finite-time time. To avoid discontinuity of (7) on $|C x|=\beta$ the function $\gamma$ is properly selected as shown below.

Theorem 1: Let the matrix $K_{\text {lin }} \in \mathbb{R}^{1 \times n}$ be the gain of a linear stabilizing feedback (2) such that Assumption 1 holds. If

- the switching surface is given by (10);

- the function $f: \mathbb{R} \times \mathbb{R}^{n} \rightarrow \mathbb{R}^{n}$ satisfies (6) with

$$
0 \leq \eta<\|P\|^{-1}
$$

and

$$
|C f(t, x)| \leq\left\{\begin{array}{cll}
\rho|\lambda||C x| & \text { if } & |C x| \geq \beta \\
\rho|\lambda| \beta & \text { if } & |C x|<\beta
\end{array}\right.
$$

where $\rho \in[0,1)$ is a scalar parameter and $P=P^{\top} \in \mathbb{R}^{n \times n}$ satisfies the Lyapunov equation

$$
\left(A+B K_{\text {lin }}\right)^{\top} P+P\left(A+B K_{\text {lin }}\right)=-2 I_{n}, \quad P \succ 0 ;
$$

- $\gamma$ is defined as follows

$$
\gamma(t, x):=\tilde{\gamma}(|C x|)=\lambda\left\{\begin{array}{cll}
|C x| & \text { if } & |C x| \geq \beta \\
\beta & \text { if } & |C x|<\beta
\end{array}\right.
$$

where $\beta>0$ is a scalar parameter; then

1) $u_{S M}=K_{\text {lin }} x$ for $|C x|>\beta$ and $C x=0$ is the only discontinuity surface of $u_{S M}$;

2) $C x=0$ is the sliding surface of the closed-loop system (1), (7) with the following reaching-time estimate

$$
T(x(0)) \leq\left\{\begin{array}{llll}
\frac{\ln \frac{|C x(0)|}{\beta}+1}{-\lambda(1-\rho)} & \text { if } & |C x(0)|>\beta, \\
\frac{|C x(0)|}{-\lambda(1-\rho) \beta} & \text { if } & |C x(0)| \leq \beta,
\end{array}\right.
$$

3) the closed-loop system (1), (7) is globally asymptotically stable.

Proof:

1) Notice that the identities (3) and (10) imply that $C\left(A+B K_{\text {lin }}\right)=\lambda C, C B=1$ and for $|C x| \geq \beta$ we have

$$
\begin{aligned}
u_{S M} & =-C A x+\lambda C x=C B K_{\text {lin }} x-C\left(A+B K_{\text {lin }}\right) x+\lambda C x \\
& =C B K_{\text {lin }} x-\lambda C x+\lambda C x=K_{\text {lin }} x .
\end{aligned}
$$

Hence, for $|C x| \geq \beta$ we derive

$$
\dot{\sigma}=C\left(A+B K_{\text {lin }}\right) x+C f(t, x)=\lambda \sigma+C f(t, x),
$$

where $\lambda<0$ by Assumption 1. The inequality (12) implies that

$$
\frac{d|\sigma|}{d t} \leq \lambda|\sigma|+\rho|\lambda||\sigma|=\lambda(1-\rho)|\sigma|<0
$$

for $|\sigma| \geq \beta$.

2) For $0<|C x|<\beta$ we have $u_{s m}(x)=-C A x+\lambda \beta \frac{C x}{|C x|}$. and

$$
\dot{\sigma}=\lambda \beta \operatorname{sign}(\sigma)+C f(t, x) .
$$

Hence, using the inequality (12) we derive

$$
\frac{d|\sigma|}{d t} \leq \lambda \beta+\rho|\lambda| \beta=\lambda(1-\rho) \beta<0
$$

for $0<|\sigma|<\beta$.

Combining (18) and (19) we conclude that the sliding mode in the surface $\sigma=0$ appears in a finite time $T(x(0))$ estimated by the formula (15).

3) For $t \geq T(x(0))$ the dynamics of the closed-loop system is described by the differential-algebraic equation (see [1])

$$
\dot{x}=A x+B u_{e q}(t)+f(t, x), \quad C x=0,
$$

where $u_{e q}(t)=-C A x-C f(t, x)$. Therefore, we have

$$
\dot{x}=(A-B C A) x+f(t, x)-B C f(t, x), \quad C x=0 .
$$

Since $C\left(A+B K_{\text {lin }}\right)=\lambda C$ and $C B=1$ then

$$
A-B C A=A-B C\left(A+B K_{l i n}-B K_{\text {lin }}\right)=A+B K_{l i n}-\lambda B C
$$

and the dynamics in the sliding mode is given by

$$
\dot{x}=\left(A+B K_{l i n}\right) x+f(t, x)-B C f(t, x), \quad C x=0 .
$$

Since $A+B K_{\text {lin }}$ is Hurwitz then there exists a symmetric positive definite solution $P \in \mathbb{R}^{n \times n}$ of the Lyapunov equation (13). Considering the Lyapunov function candidate

$$
V=x^{\top} P x
$$

we derive

$$
\begin{gathered}
\dot{V}=2 x^{\top} P\left(A+B K_{\text {lin }}\right) x+2 x^{\top} P(f(t, x)-B C f(t, x)) \leq \\
-2\|x\|^{2}+2\|x\|\|P(f(t, x)-B C f(t, x))\| \leq \\
-2\|x\|(\|x\|-\|P\| \cdot\|f(t, x)-B C f(t, x)\|)<0
\end{gathered}
$$

provided that $\|f(t, x)-B C f(t, x)\|<\|P\|^{-1}\|x\|$. The proof is complete.

The sliding mode controller (7), (14) can be rewritten as a linear feedback with the state dependent gain:

$$
u_{S M}=K_{S M}(|C x|) x \quad \text { for } \quad C x \neq 0,
$$

where $K_{S M}:(0,+\infty) \rightarrow \mathbb{R}^{1 \times n}$ is defined as follows

$$
K_{S M}(\varphi)=K_{\text {nom }}+\frac{\tilde{\gamma}(\varphi)}{\varphi} C, \quad \varphi>0 .
$$

Below we use this representation of the sliding mode controller in order to introduce a chattering reduction scheme. Notice that the sliding mode control designed by the latter theorem coincides with the linear feedback for $|C x| \geq \beta$. For $\beta$ tending to zero the original linear feedback is recovered. From a theoretical point of view, larger the $\beta$, larger the magnitude of the matched perturbations to be rejected (see, the formula (12)). In practice, large $\beta$ may invoke large chattering magnitude, so tuning the parameter $\beta$ would allow us to upgrade a linear control to a sliding mode one and prevent a degradation of the control accuracy due to the chattering phenomenon.

Remark 1: By Assumption 1, the suggested transformation of the linear feedback to SMC is possible if the matrix $A+B K_{l i n}$ has a real negative eigenvalue. If this matrix has only complex pairs of eigenvalues, another scheme of the upgrade has to be developed. In this case, results similar to Theorem 1 can be developed for the multi-input system. 
Remark 2: Any real negative eigenvalue of the matrix $A+B K_{\text {lin }}$ satisfying Assumption 1 can be selected for the upgrade. However, in practice this selection may impact the improvement of the control quality of the obtained SMC. The best option in this case is to compare the corresponding sliding mode controllers on the experiments and select the best one. If such a comparison is not possible the largest negative eigenvalue should be selected among others.

\section{A SCHEME OF PRACTICAL IMPLEMENTATION}

The classical idea for chattering reduction of SMC is to replace the sign multifunction by a piecewise-linear saturation function, and, next, to tune its width parameter[1]. This widely used engineering trick is known, however, it decreases the closed-loop accuracy since it destroys the sliding-mode phase. This is what is done below, taking into account the structure of the proposed SMC.

As shown above the sliding mode controller (7), (14) can be interpreted as a linear feedback

$$
u_{S M}=K_{S M}(|C x|) x
$$

with the state-dependent gain $K_{S M}(|C x|)$ given by (20), which tends to $\infty$ as $|C x| \rightarrow 0$. The infinite gain in the linear controller contributes to the so-called chattering phenomenon [1], [7]. The simplest approach to reduce the chattering is to bound the gain $K_{S M}(|C x|)$ close to singularity points. Let us introduce the following saturation function

$$
\operatorname{sat}_{\delta, \beta}(\varphi)=\left\{\begin{array}{llc}
\delta & \text { if } & \varphi<\delta \\
\varphi & \text { if } & \delta \leq \varphi \leq \beta \\
\beta & \text { if } & \varphi>\beta
\end{array}\right.
$$

To avoid the infinite gain in the explicit discretization of the sliding mode controller, we re-define it as follows

$$
\tilde{u}(x)=K_{S M}\left(\operatorname{sat}_{\delta, \beta}(|C x|)\right) x,
$$

where $\beta>0$ is defined in the formula (14) and $\delta \in(0, \beta)$ is a tuning parameter. Obviously, $\delta=0$ corresponds to the original sliding-mode controller, but for $\delta=\beta$ it follows that

$$
K_{S M}\left(\operatorname{sat}_{\beta, \beta}\left(\left|C x_{k}\right|\right)\right)=K_{\text {lin }}
$$

i.e, the proposed controller becomes the linear feedback in the limit case.

Therefore, we can upgrade a linear controller to sliding mode controller. To avoid the degradation of the control quality, the following procedure can be utilized

1) design the parameters of the sliding mode controller using Theorem 1 for some $\beta>0$;

2) implement the control law (22) in a digital device with $\delta=\beta$ (linear feedback);

3) decrease the parameter $\delta$ while the control quality is improving.

The control quality in the latter scheme can be estimated through numerical simulations or performing experiments on a setup. It may include various indexes, which correspond to control precision, energetic optimality, chattering effects, etc.

\section{EXPERIMENT: ROTARY INVERTED PENDULUM}

The platform QUBE-Servo 2 of Quanser is used for the experiment, and it is depicted in Fig. 2 together with the convention of the sign for the angles $\theta$ and $\alpha$, respectively for the rotary arm and the pendulum arm.
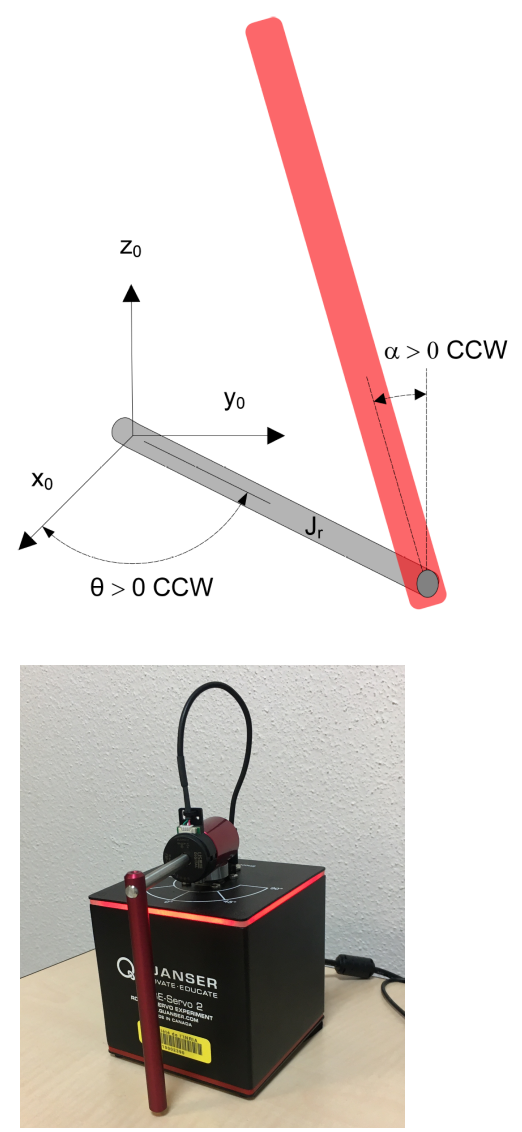

Fig. 2. Schematics and photo of the rotary inverted pendulum.

The platform is supported with both a swing-up controller and a linear stabilizing controller realized in Matlab, and we modify only the linear stabilizing controller. The conceptual scheme of whole controller architecture is depicted in Fig. 3 . The switching activity from the energy swing-up and the stabilizing controllers occurs when $|\alpha|<20 \mathrm{deg}$. The linear model of the pendulum system can be expressed in state-space form as:

$$
\dot{x}(t)=A x(t)+B u(t)
$$

where $x(t)=(\theta(t), \alpha(t), \dot{\theta}(t), \dot{\alpha}(t))^{\top} \in \mathbb{R}^{4}$ is the state vector, $u(t) \in \mathbb{R}$ is the control signal, $A \in \mathbb{R}^{4 \times 4}, B \in \mathbb{R}^{4 \times 1}$. The matrices of the linear systems are given by the manufacturer and they are

$A=\left(\begin{array}{cccc}0 & 0 & 1 & 0 \\ 0 & 0 & 0 & 1 \\ 0 & 149.2751 & -0.0104 & 0 \\ 0 & 261.6091 & -0.0103 & 0\end{array}\right), B=\left(\begin{array}{c}0 \\ 0 \\ 49.7275 \\ 49.1493\end{array}\right)$.

The parameters of the experimental platform are also given by the manufacturer and they are listed in the Table I. The 


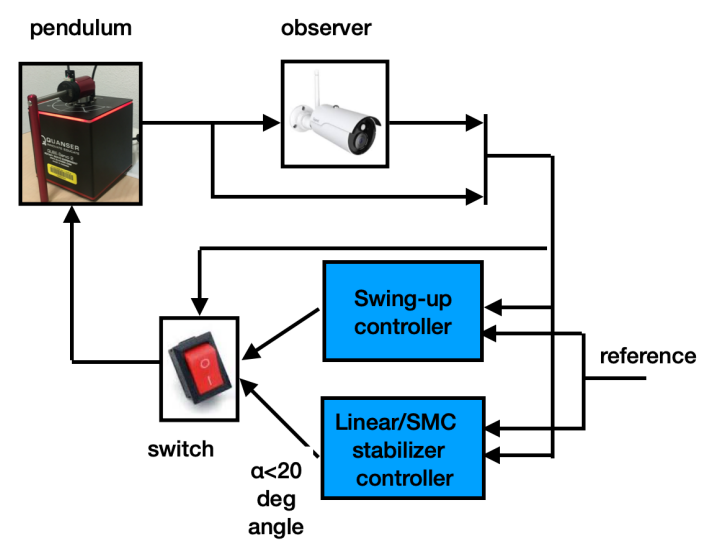

Fig. 3. Conceptual scheme of the controller for the rotary inverted pendulum.

TABLE I

Parameters of THE QUBE-Servo 2

\begin{tabular}{|l|l|}
\hline Parameter & Value \\
\hline$m_{p}$ & $0.024 \mathrm{Kg}$ \\
\hline$L_{p}$ & $0.129 \mathrm{~m}$ \\
\hline$J_{p}$ & $3.3 \times 10^{-5} \mathrm{Kg} \cdot \mathrm{m}^{2}$ \\
\hline$D_{p}$ & $0.0015 \mathrm{~N} . \mathrm{m} . \mathrm{s} / \mathrm{rad}$ \\
\hline$L_{r}$ & $0.085 \mathrm{~m}$ \\
\hline$J_{r}$ & $5.7 \times 10^{-5} \mathrm{Kg} \cdot \mathrm{m}^{2}$ \\
\hline$D_{r}$ & $0.0005 \mathrm{~N} . \mathrm{m} . \mathrm{s} / \mathrm{rad}$ \\
\hline$g$ & $9.81 \mathrm{~m} / \mathrm{s}^{2}$ \\
\hline
\end{tabular}

experiments are carried out with a fixed sampling time $T=$ $0.002 s$, which is given by the encoder of the device and cannot be changed, for a total experimental time of $20 \mathrm{~s}$. The gains of the linear proportional controllers are given by the manufacturer, and they are already optimally tuned:

$$
K_{\text {lin }}=[2,-35,1.5,-3] .
$$

The matrix $A+B K_{l i n}$ has the following eigenvalues

$$
\begin{gathered}
\lambda_{1}=-48.2522 ; \lambda_{2}=-11.4050+0.4427 i ; \\
\lambda_{3}=-11.4050-0.4427 i ; \lambda_{4}=-1.8048 .
\end{gathered}
$$

Two of them are real negative and can be utilized for the upgrade. Table II shows the effect of the two eigenvalues on the upgraded SMC. The experiments showed that the choice of $\lambda=\lambda_{4}$ produces a better precision with less control effort. For this reason, in the subsequent study we use $\lambda=\lambda_{4}$ for the computation of the SMC and its comparison with the original linear algorithm.

TABLE II

DEPENDENCE OF THE CONTROL QUALITY ON THE SELECTION OF THE EIGENVALUE FOR THE SMC DESIGN

\begin{tabular}{|l|l|l|l|l|l|}
\hline & $\left\|e_{\theta}\right\|_{L^{2}}$ & $\left\|e_{\alpha}\right\|_{L^{2}}$ & $\left\|\dot{e}_{\theta}\right\|_{L^{2}}$ & $\left\|\dot{e}_{\alpha}\right\|_{L^{2}}$ & $\|u\|_{L^{2}}$ \\
\hline$\lambda_{1}=-48.2522$ & 0.25045 & 0.02940 & 0.37449 & 0.31906 & 1.51214 \\
\hline$\lambda_{4}=-1.8048$ & 0.16965 & 0.03042 & 0.32122 & 0.27482 & 1.20613 \\
\hline \hline & $\left\|e_{\theta}\right\|_{L^{\infty}}$ & $\left\|e_{\alpha}\right\|_{L^{\infty}}$ & $\left\|\dot{e}_{\theta}\right\|_{L^{\infty}}$ & $\left\|\dot{e}_{\alpha}\right\|_{L^{\infty}}$ & $\|u\|_{L^{\infty}}$ \\
\hline$\lambda_{1}=-48.2522$ & 0.11074 & 0.01534 & 0.37825 & 0.23308 & 1.20076 \\
\hline$\lambda_{4}=-1.8048$ & 0.07878 & 0.01227 & 0.24802 & 0.20245 & 0.97999 \\
\hline
\end{tabular}

TABLE III

$L^{\infty}$ AND $L^{2}$ NORMS OF THE CONTROLLERS IN THE FIRST SCENARIO.

\begin{tabular}{|l|l|l|l|l|l|}
\hline & $\left\|e_{\theta}\right\|_{L^{2}}$ & $\left\|e_{\alpha}\right\|_{L^{2}}$ & $\left\|\dot{e}_{\theta}\right\|_{L^{2}}$ & $\left\|\dot{e}_{\alpha}\right\|_{L^{2}}$ & $\|u\|_{L^{2}}$ \\
\hline Linear & 0.31839 & 0.03287 & 0.34711 & 0.26787 & 1.19112 \\
\hline SMC & 0.16965 & 0.03042 & 0.32122 & 0.27482 & 1.20613 \\
\hline \hline & $\left\|e_{\theta}\right\| \|_{L^{\infty}}$ & $\left\|e_{\alpha}\right\|_{L^{\infty}}$ & $\left\|\dot{e}_{\theta}\right\| \|_{L^{\infty}}$ & $\left\|\dot{e}_{\alpha}\right\| L_{L^{\infty}}$ & $\|u\|_{L^{\infty}}$ \\
\hline Linear & 0.13929 & 0.01534 & 0.40183 & 0.19115 & 0.95631 \\
\hline SMC & 0.07878 & 0.01227 & 0.24802 & 0.20245 & 0.97999 \\
\hline
\end{tabular}

TABLE IV

$L^{\infty}$ AND $L^{2}$ NORMS OF THE CONTROLLERS IN THE SECOND SCENARIO.

\begin{tabular}{|l|l|l|l|l|l|}
\hline & $\left\|e_{\theta}\right\|_{L^{2}}$ & $\left\|e_{\alpha}\right\|_{L^{2}}$ & $\left\|\dot{e}_{\theta}\right\|_{L^{2}}$ & $\left\|\dot{e}_{\alpha}\right\|_{L^{2}}$ & $\|u\|_{L^{2}}$ \\
\hline Linear & 1.02338 & 0.06496 & 1.38133 & 0.44407 & 1.43855 \\
\hline SMC & 0.95564 & 0.07315 & 1.63003 & 0.52998 & 1.60226 \\
\hline \hline & $\left\|e_{\theta}\right\|_{L^{\infty}}$ & $\left\|e_{\alpha}\right\|_{L^{\infty}}$ & $\left\|\dot{e}_{\theta}\right\|_{L^{\infty}}$ & $\left\|\dot{e}_{\alpha}\right\|_{L^{\infty}}$ & $\|u\|_{L^{\infty}}$ \\
\hline Linear & 1.19282 & 0.06136 & 2.01917 & 1.32961 & 2.74149 \\
\hline SMC & 1.22350 & 0.06443 & 2.31936 & 1.32893 & 2.86381 \\
\hline
\end{tabular}

Three scenarios are tested: in the first one the reference signals are given by $\alpha_{\text {ref }}=0 \mathrm{rad}, \theta_{\text {ref }}(t)=0.3 \sin (t) \mathrm{rad}$; in the second one $\theta_{\text {ref }}=0 \mathrm{rad}$ for $t \leq 5 \mathrm{~s}, \theta_{\text {ref }}=0.6 \mathrm{rad}$ for $5<t \leq 10 s, \theta_{\text {ref }}=-0.6 \mathrm{rad}$ for $10<t \leq 15 s, \theta_{\text {ref }}=0 \mathrm{rad}$ for $t>15 \mathrm{~s}$; in the third one $\theta_{\text {ref }}=0 \mathrm{rad}$ for all $t$. The derivatives are not available directly from the output, so a linear differentiator is used, having transfer function $F(s)=$ $50 s /(s+50)$. The observer dynamics should be much faster than the reaching time of the sliding mode. The aim of the controllers is to minimize the error in $\theta$. The swingup controller is active during approximately $3 \mathrm{sec}$. Thus a comparison of the performances of the linear and of the sliding mode control algorithms is made using the $L^{2}$ and $L^{\infty}$ norms of both the input $u$ and of the tracking errors:

$$
e_{\theta}=\theta-\theta_{r e f}, e_{\alpha}=\alpha-\alpha_{r e f}, \dot{e}_{\theta}=\dot{\theta}-\dot{\theta}_{r e f}, \dot{e}_{\alpha}=\dot{\alpha}-\dot{\alpha}_{r e f}
$$

on the time interval $[3,20]$ seconds.

Tables III, IV, V show the performances of the two controllers, after tuning $\delta=0.65, \beta=1$ for the SMC. The smaller $\delta$, the smaller the error in $\theta$, but with more accentuated control effort, and vice-versa. Therefore, the additional parameter $\delta$ must be carefully tuned depending on the constraints of the application. Figures 4, 5, 6 depict the transients of the error $\left|\theta_{\text {ref }}-\theta\right|$ and of the input $u$ for the linear controller and SMC. The SMC achieves about $40 \%$ better precision in the first scenario than the linear controller The improvement for the third scenario is about $20 \%$. The precision for the second scenario is obviously improved in the steady states (for the time intervals $[6,10],[11,15]$ and $[16,20])$, but the response to a change of the set-point (a step response) is similar for both controllers (see Fig. 5).

\section{CONCLUSION}

In this work, a procedure to upgrade the linear controller to a sliding mode one is introduced. It modifies the linear feedback in a certain zone of the state space and transforms it to a sliding mode controller. Based on the structure of the obtained SMC, a special scheme of its practical implementation preventing a degradation of the control quality is proposed. 
TABLE V

$L^{\infty}$ AND $L^{2}$ NORMS OF THE CONTROLLERS IN THE THIRD SCENARIO.

\begin{tabular}{|l|c|c|c|c|c|}
\hline & $\left\|e_{\theta}\right\|_{L^{2}}$ & $\left\|e_{\alpha}\right\|_{L^{2}}$ & $\left\|\dot{e}_{\theta}\right\|_{L^{2}}$ & $\left\|\dot{e}_{\alpha}\right\|_{L^{2}}$ & $\|u\|_{L^{2}}$ \\
\hline Linear & 0.18630 & 0.01442 & 0.26178 & 0.20637 & 0.73179 \\
\hline SMC & 0.13160 & 0.01637 & 0.26674 & 0.24158 & 0.88139 \\
\hline \hline & $\left\|e_{\theta}\right\|_{L^{\infty}}$ & $\left\|e_{\alpha}\right\|_{L^{\infty}}$ & $\left\|\dot{e}_{\theta}\right\|_{L^{\infty}}$ & $\left\|\dot{e}_{\alpha}\right\|_{L^{\infty}}$ & $\|u\|_{L^{\infty}}$ \\
\hline Linear & 0.15033 & 0.01227 & 0.42319 & 0.20171 & 0.68464 \\
\hline SMC & 0.08897 & 0.00921 & 0.36717 & 0.23925 & 0.74092 \\
\hline
\end{tabular}
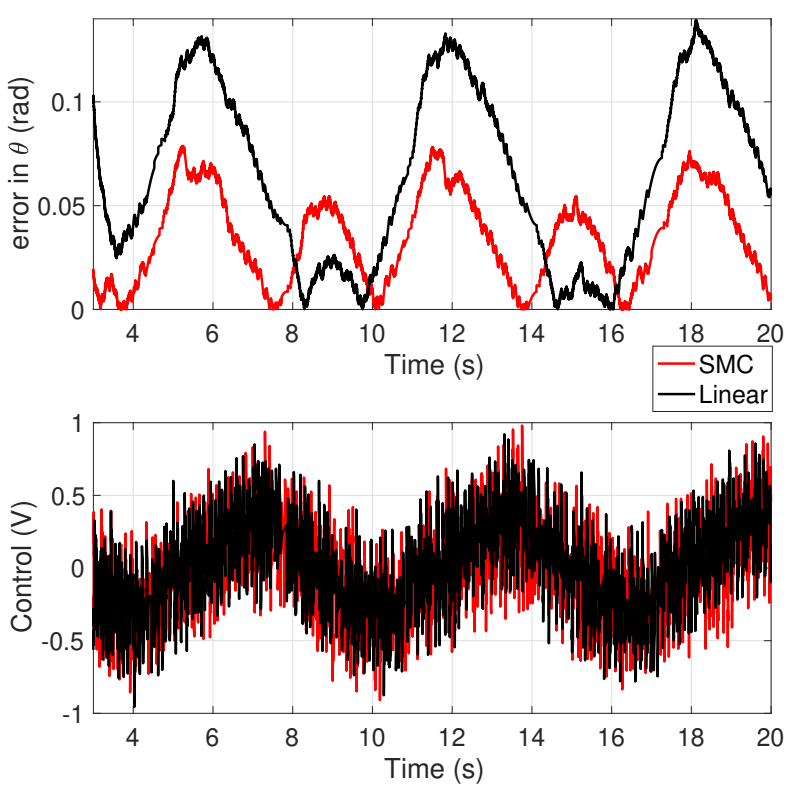

Fig. 4. Comparison between Linear and SMC controllers in the first scenario.
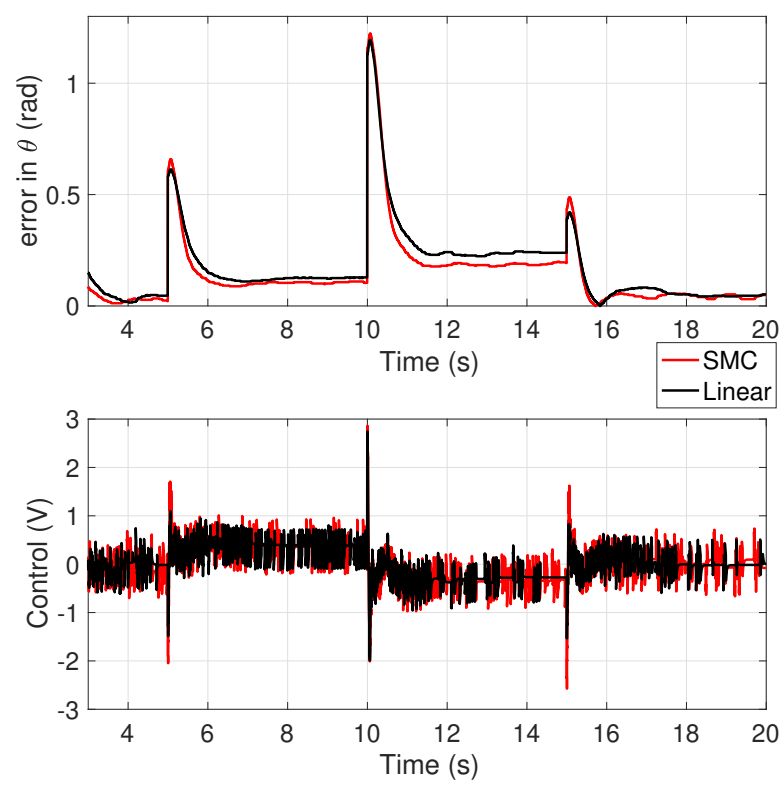

Fig. 5. Comparison between Linear and SMC controllers in the second scenario.
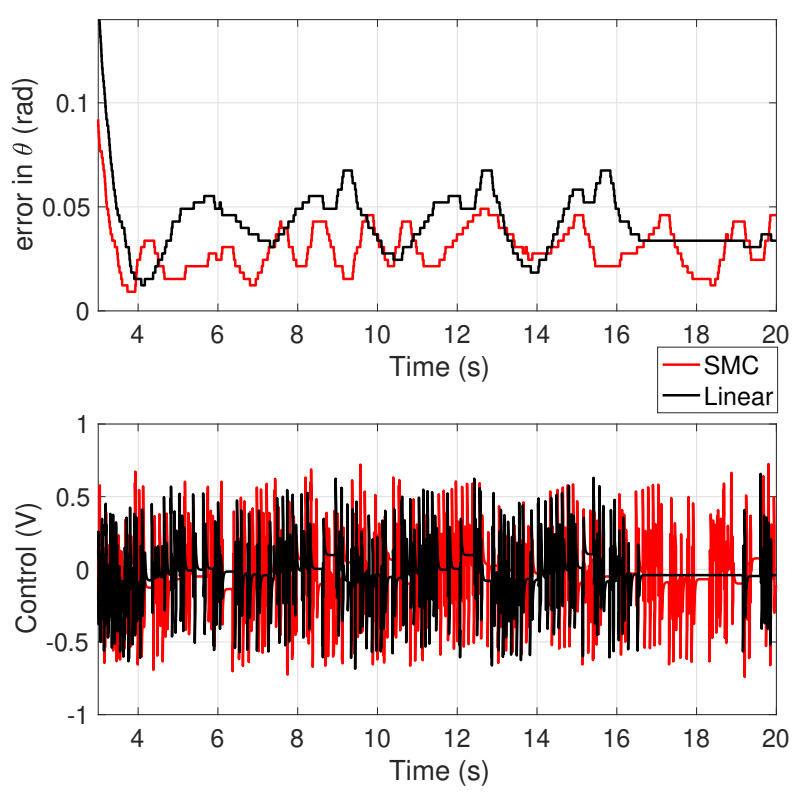

Fig. 6. Comparison between Linear and SMC controllers in the third scenario.

The theoretical results are supported by experiments with inverted rotary pendulum. The obtained experimental results show that the upgrading strategy improves the performance of the given linear controller, while keeping a comparable control effort for both inputs.

This approach presents a promising research direction for further investigations.

\section{REFERENCES}

[1] V. I. Utkin, Sliding Modes in Control Optimization. Berlin: SpringerVerlag, 1992.

[2] C. Edwards and S. Spurgeon, Sliding Mode Control: Theory and Applications. Taylor and Francis, 1998.

[3] Y. Shtessel, C. Edwards, L. Fridman, and A. Levant, Sliding Mode Control and Observation. Birkhauser, 2014.

[4] A. Levant, "Sliding order and sliding accuracy in sliding mode control," International Journal of Control, vol. 58, no. 6, pp. 1247-1263, 1993.

[5] A. Levant, "Chattering analysis," IEEE Transactions on Automatic Control, vol. 55, no. 6, pp. 1380-1389, 2010.

[6] I. Boiko, Discontinuous control systems : frequency-domain analysis and design. Boston : Birkhauser, 2009.

[7] V. Acary and B. Brogliato, "Implicit euler numerical scheme and chattering-free implementation of sliding mode systems," Systems \& Control Letters, vol. 59, no. 5, pp. 284-293, 2010.

[8] F. Plestan, Y. Shtessel, V. Bregeault, and A. Poznyak, "New methodologies for adaptive sliding mode control," International Journal of Control, vol. 83, no. 9, pp. 1907-1919, 2010.

[9] V. Utkin, A. Poznyak, Y. Orlov, and A. Polyakov, "Conventional and high order sliding mode control," Journal of the Franklin Institute, vol. 357, pp. 10244-10261, Oct. 2020.

[10] U. Pérez-Ventura and L. Fridman, "When is it reasonable to implement the discontinuous sliding-mode controllers instead of the continuous ones? frequency domain criteria," International Journal of Robust and Nonlinear Control, vol. 29, no. 3, pp. 353-374, 2019.

[11] Y. Bavafa-Toosi, Introduction to Linear Control Systems. Elsevier, 2017.

[12] S. Boyd, E. Ghaoui, E. Feron, and V. Balakrishnan, Linear Matrix Inequalities in System and Control Theory. Philadelphia: SIAM, 1994.

[13] A. Poznyak, A. Polyakov, and V. Azhmyakov, Attractive Ellipsoids in Robust Control. Birkhauser, 2014. 